Figure 1

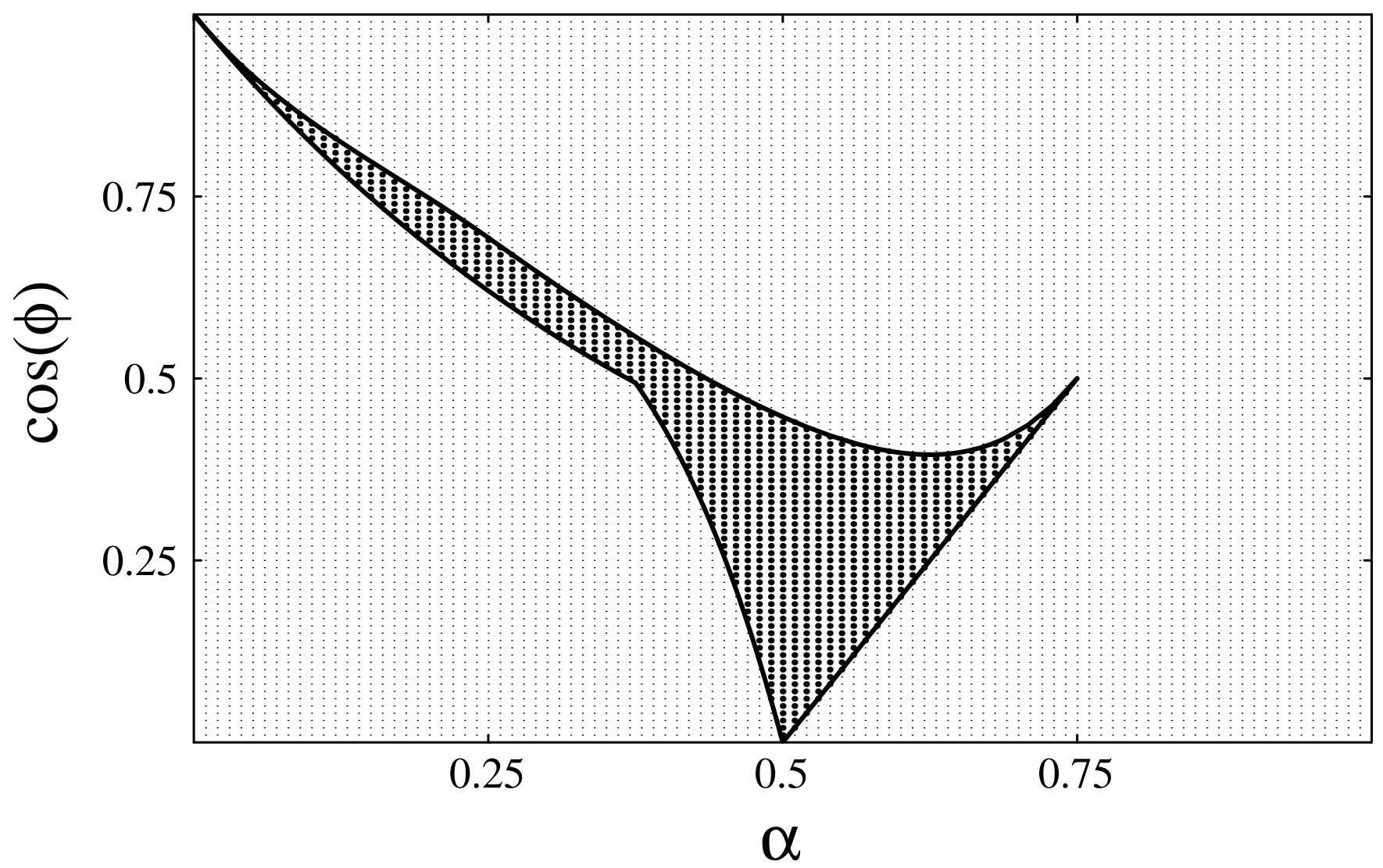


Figure 2a

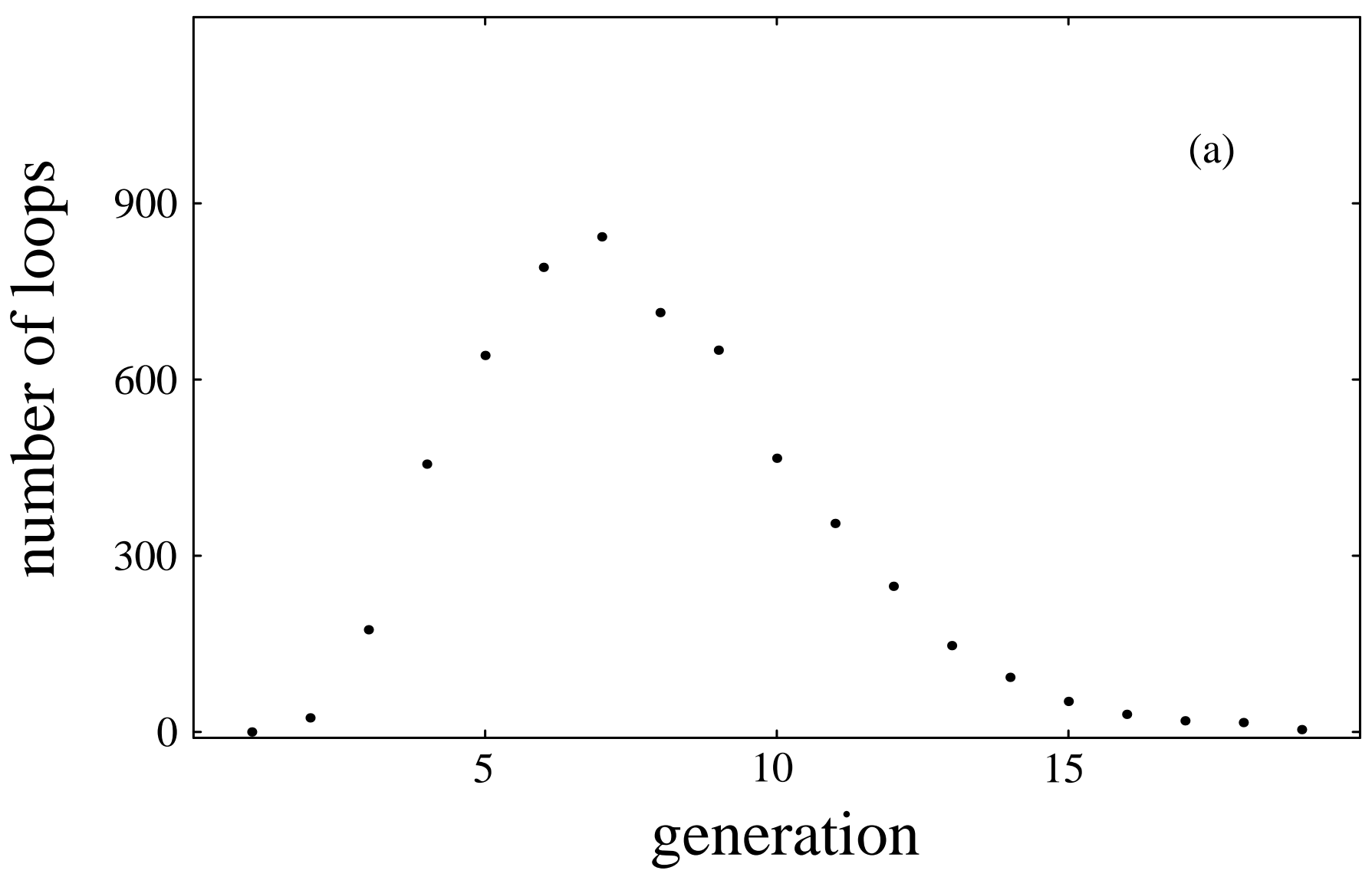


Figure $2 b$

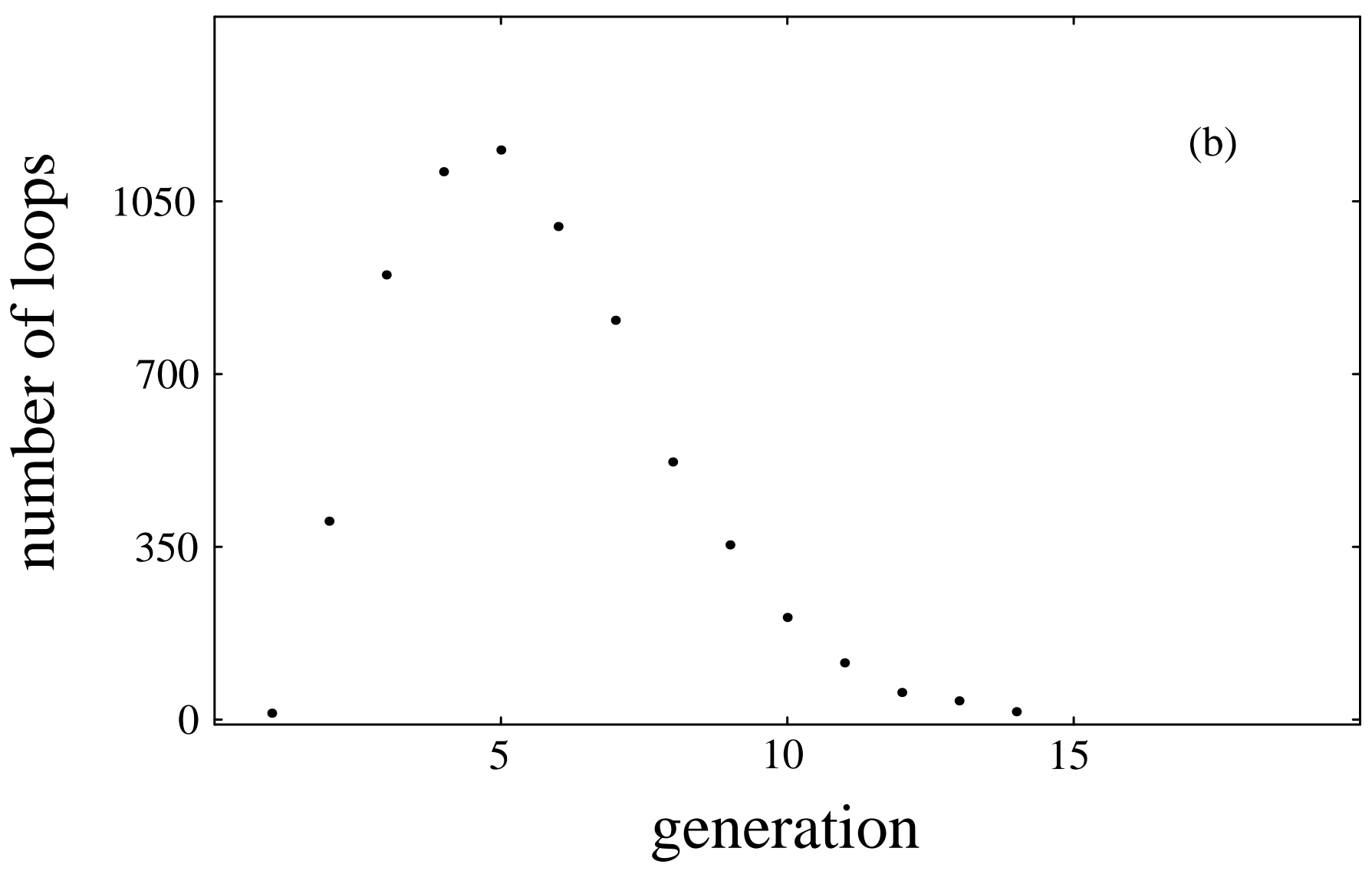


Figure 2c

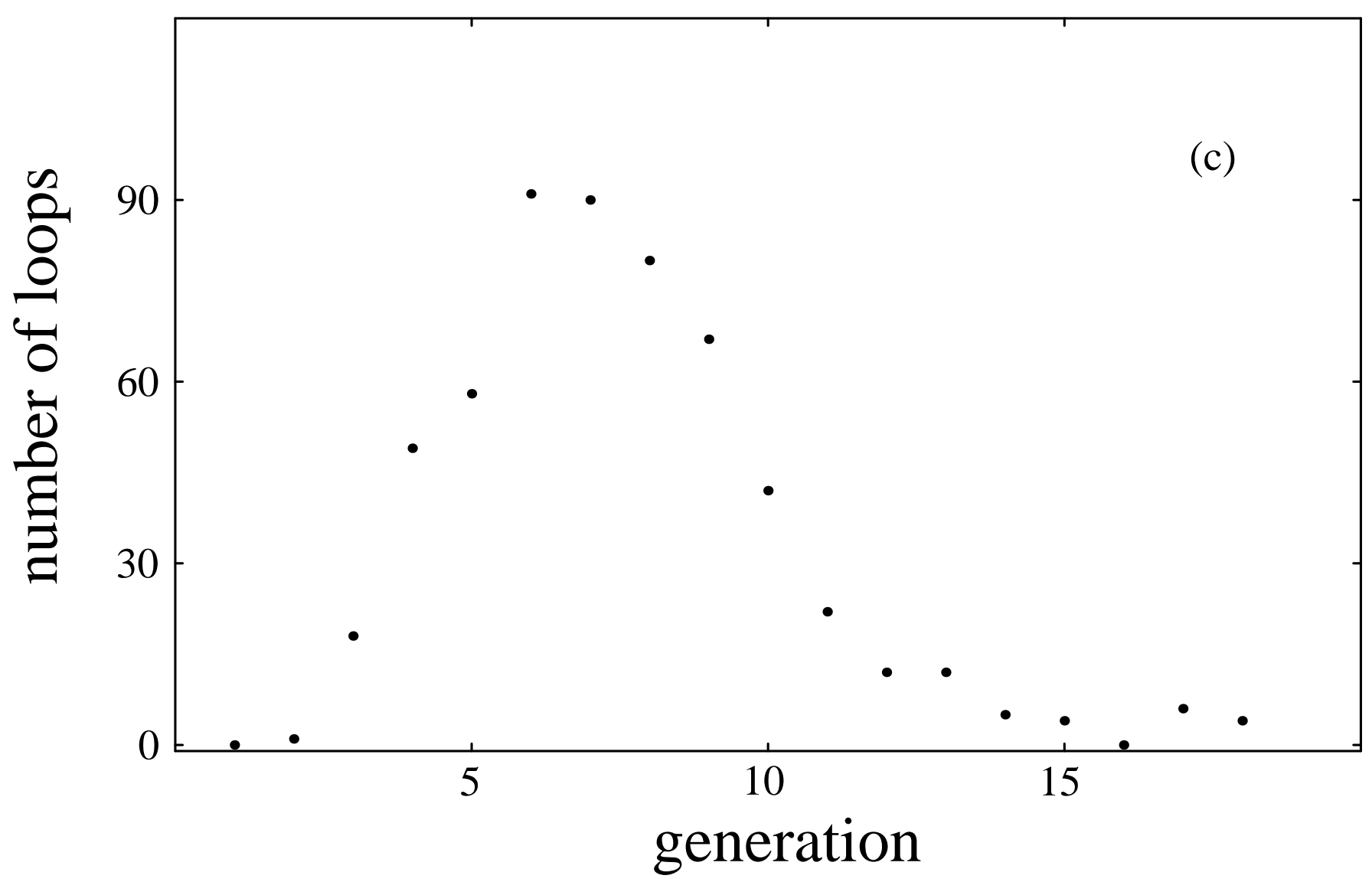


Figure 2d

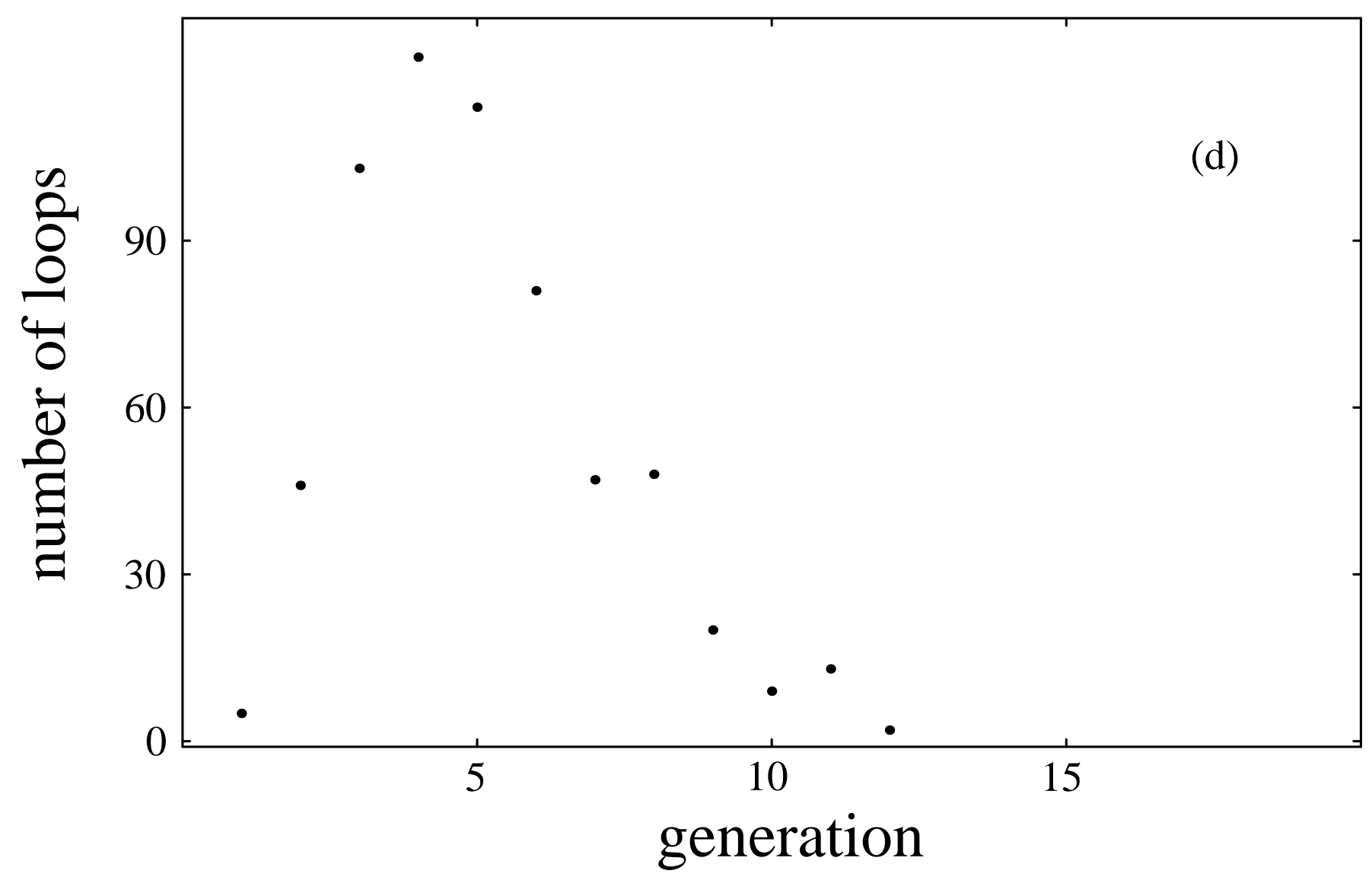


Figure 3a

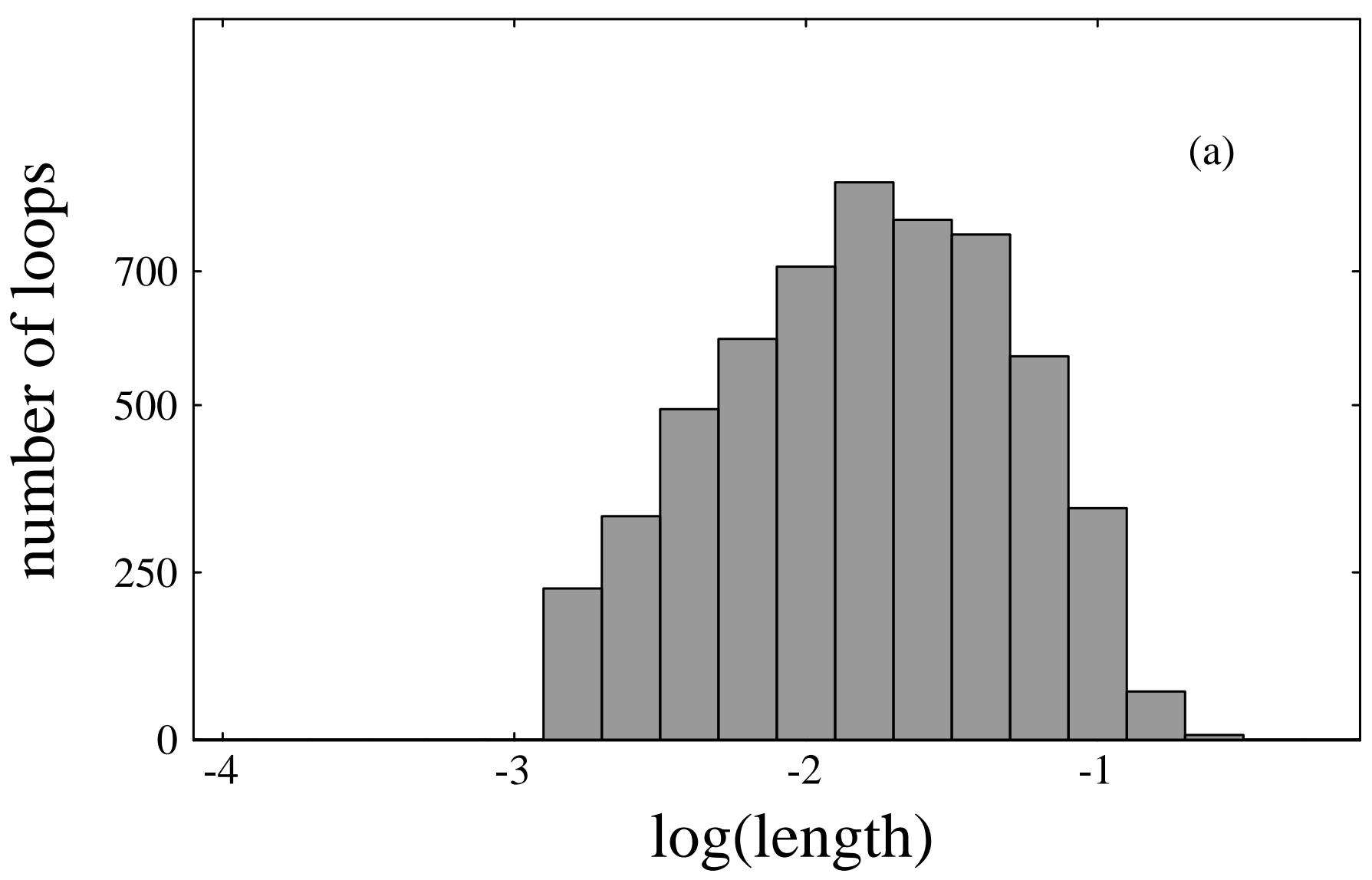


Figure $3 b$

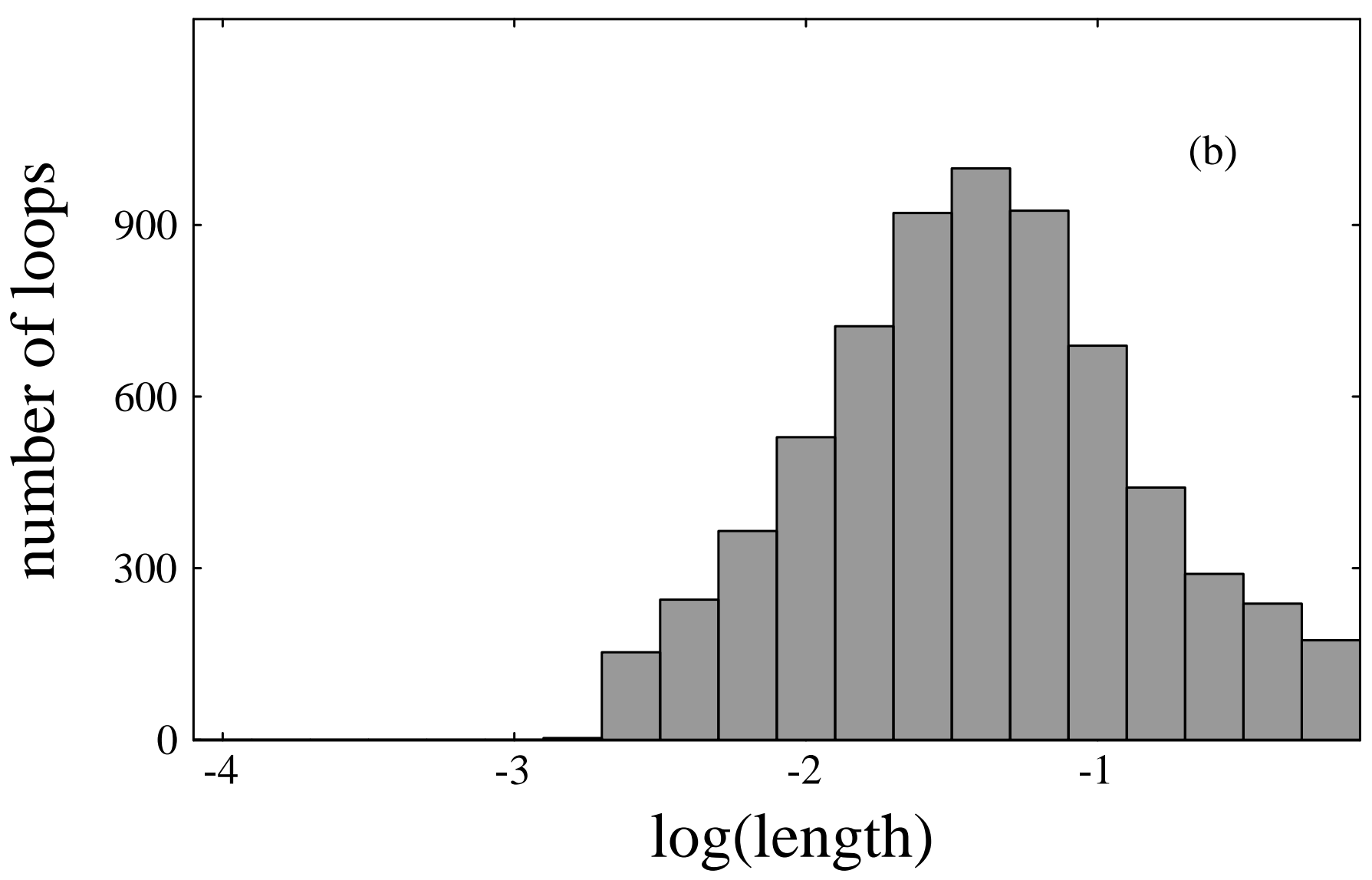


Figure 3c

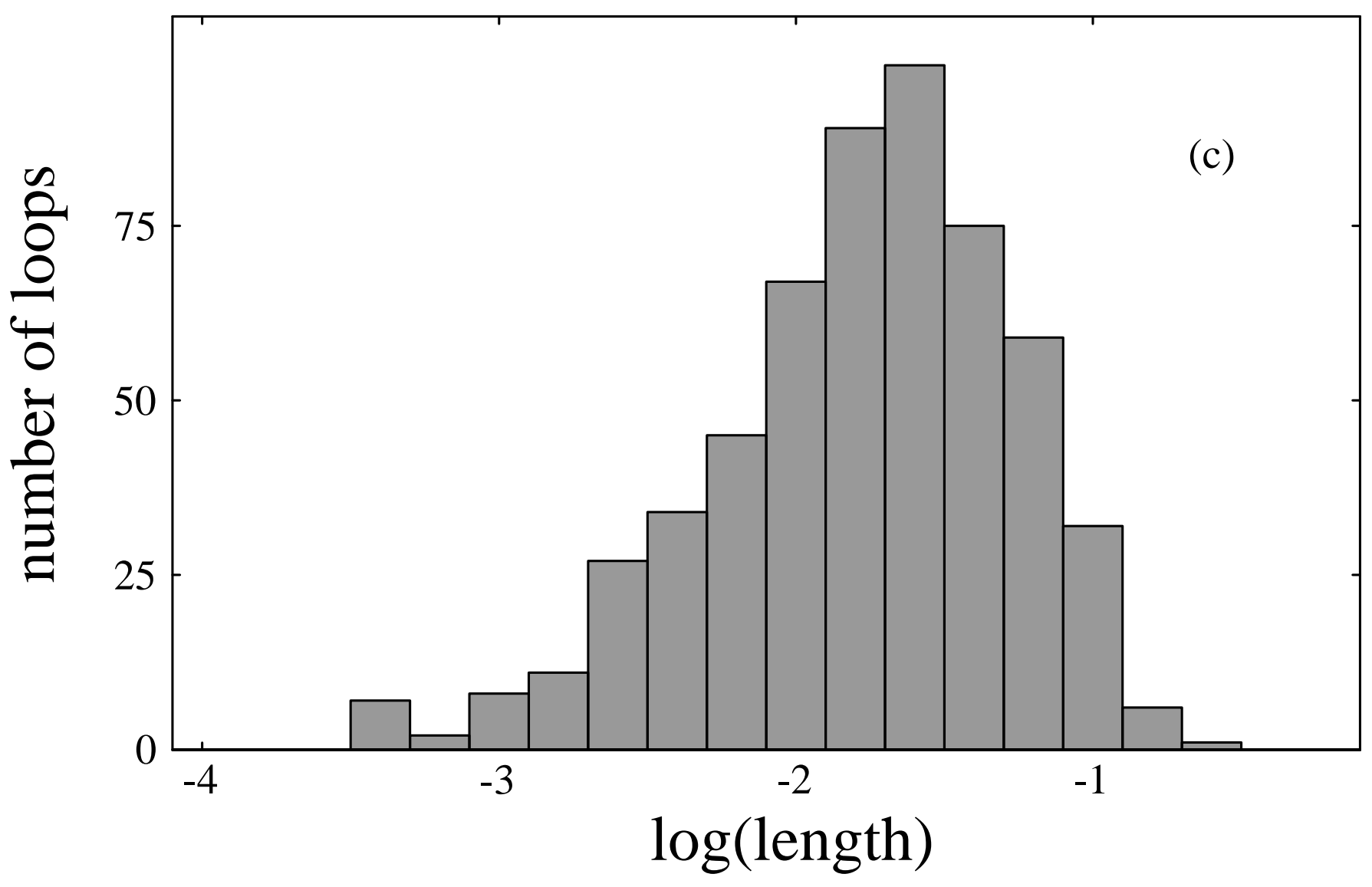


Figure 3d

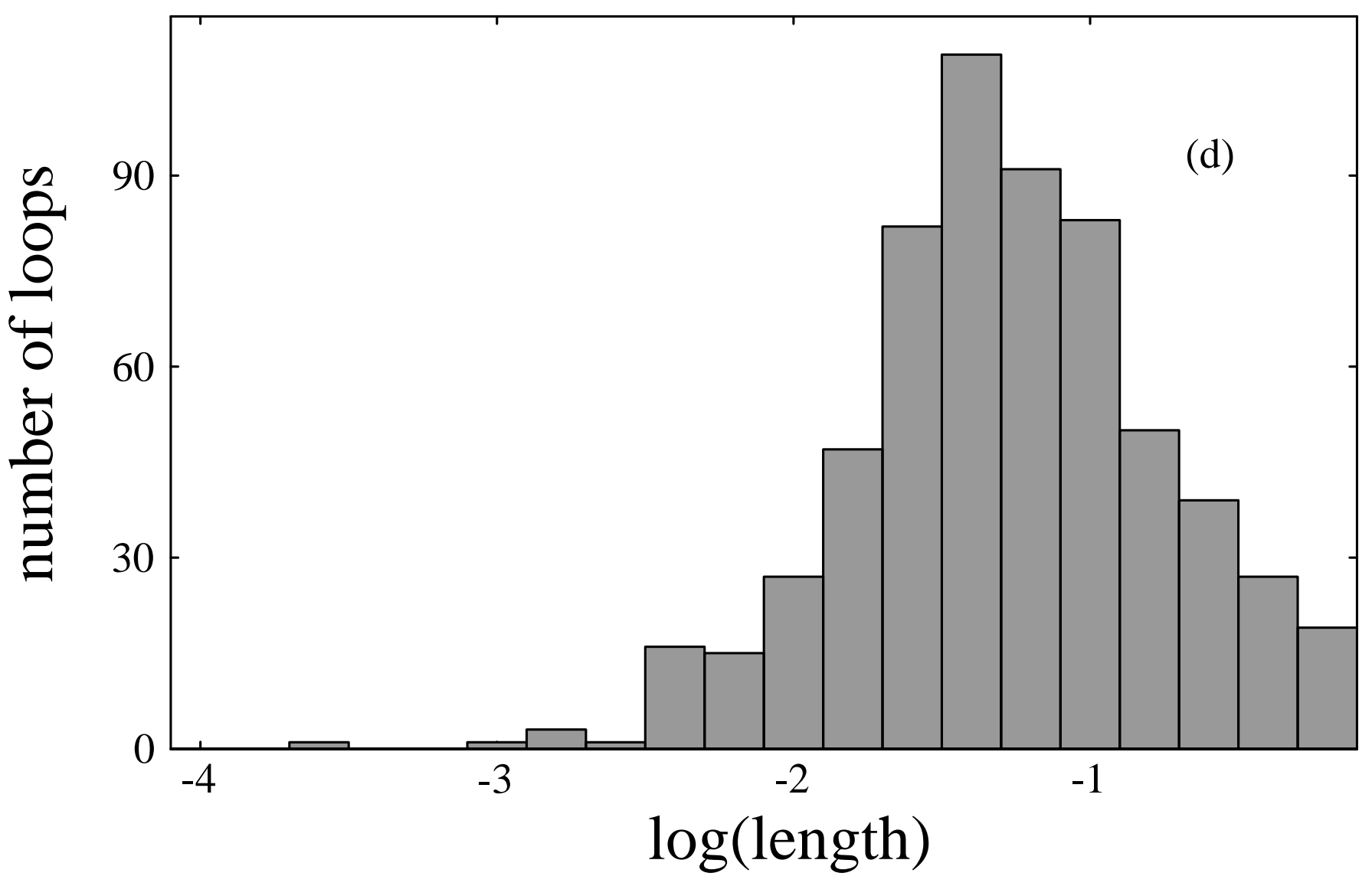


Figure 4a

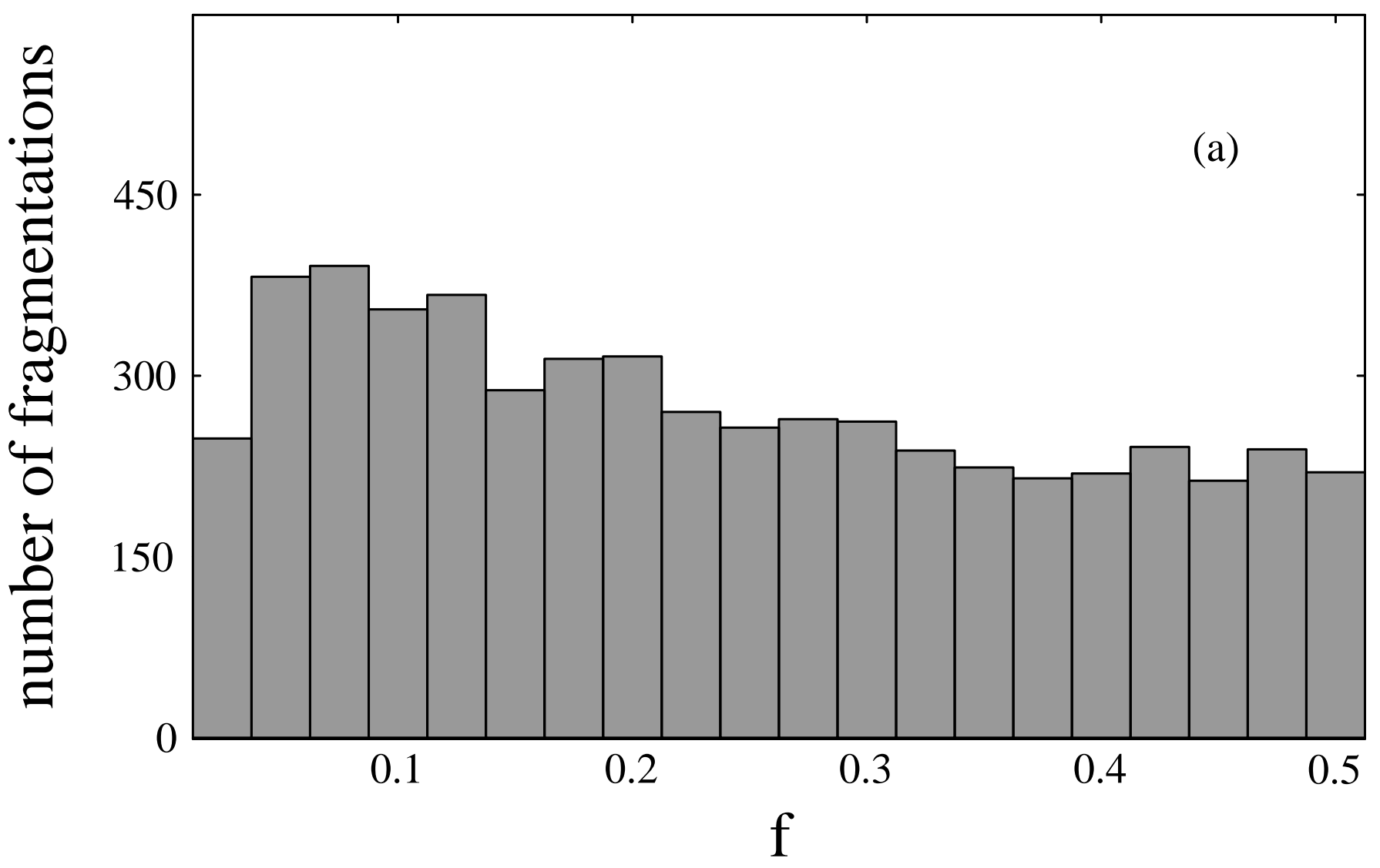


Figure $4 b$

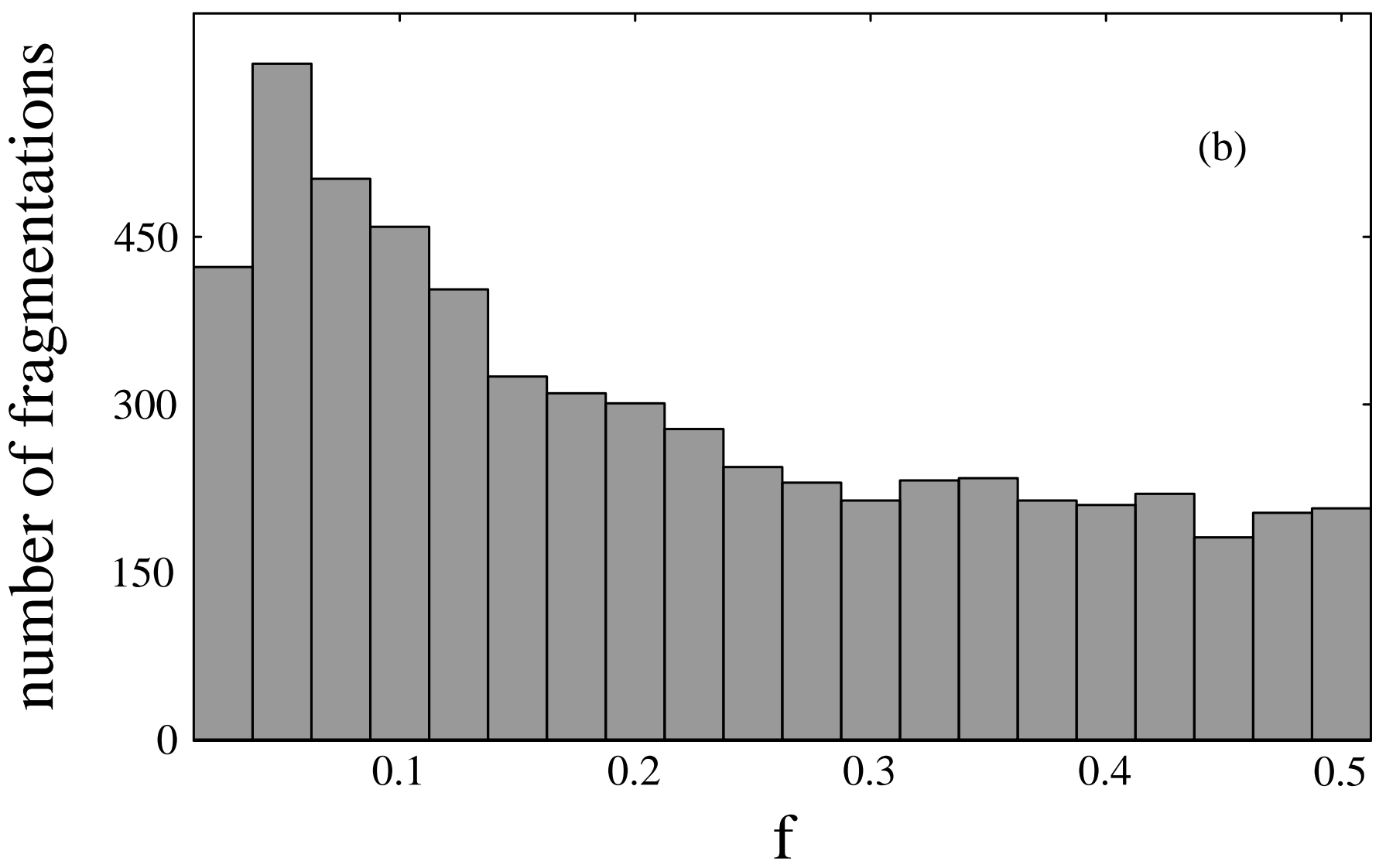


Figure 4c

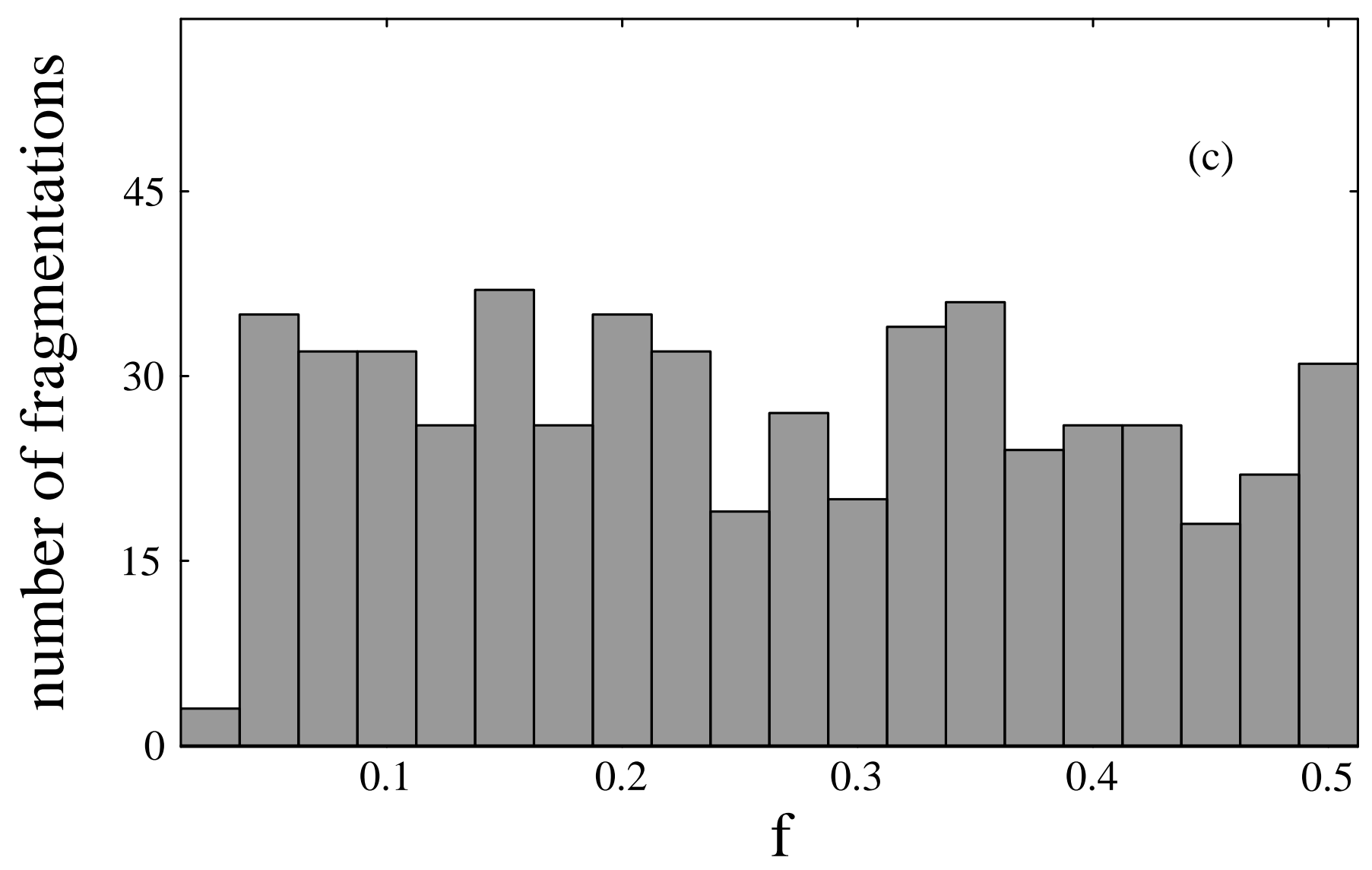


Figure 4d

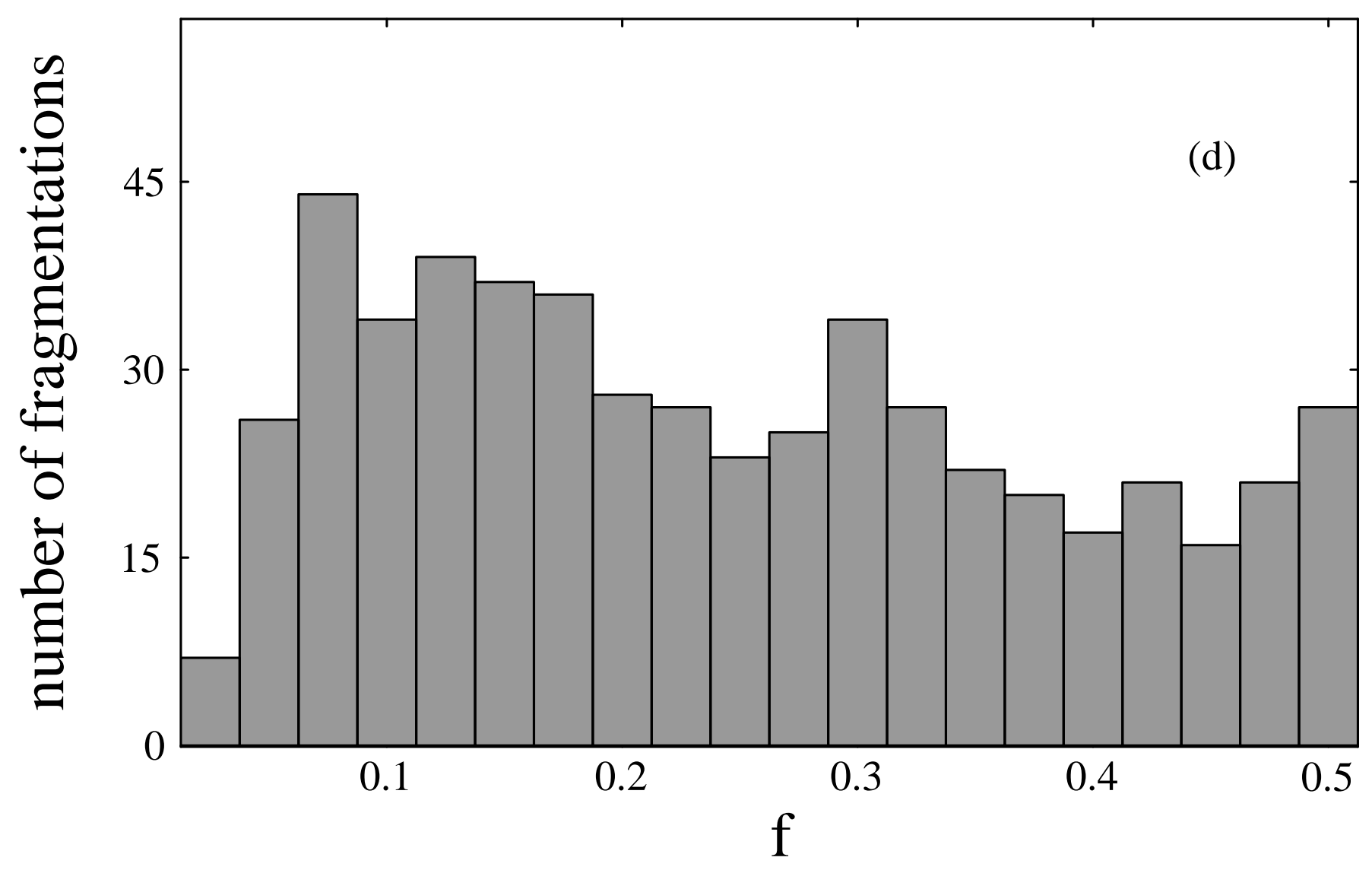


WISC-MILW-95-TH-11

\title{
GRAVITATIONAL RADIATION FROM REALISTIC COSMIC STRING LOOPS
}

\author{
Paul Casper \\ Department of Physics, University of Wisconsin-Mitwaukee \\ P.O. Box 413, Mitwaukee, Wisconsin 53201, U.S.A. \\ email: pcasper@dirac.phys.uwm.edu \\ Bruce Allen \\ Department of Physics, University of Wisconsin - Mitwaukee \\ P.O. Box 413, Mitwaukee, Wisconsin 53201, U.S.A. \\ email: ballen@dirac.phys.uwm.edu
}

(May 11, 1995)

\begin{abstract}
We examine the rates at which energy and momentum are radiated into gravitational waves by a large set of realistic cosmic string loops. The string loops are generated by numerically evolving parent loops with different initial conditions forward in time until they self-intersect, fragmenting into two child loops. The fragmentation of the child loops is followed recursively until only non-self-intersecting loops remain. The properties of the final non-selfintersecting loops are found to be independent of the initial conditions of the parent loops. We have calculated the radiated energy and momentum for a total of 11,625 stable child loops. We find that the majority of the final loops do not radiate significant amounts of spatial momentum. The velocity gained due to the rocket effect is typically small compared to the center-of-mass velocity of the fragmented loops. The distribution of gravitational radiation rates in the center of mass frame of the loops, $\gamma^{0} \equiv\left(G \mu^{2}\right)^{-1} \Delta E / \Delta \tau$, is strongly peaked in the range $\gamma^{0}=45-55$, however there are no loops found with $\gamma^{0}<40$. Because the radiated spatial momentum is small, the distribution of gravitational radiation rates appears roughly the same in any reference frame. We conjecture that in the center-of-mass frame there is a lower bound $\gamma_{\min }^{0}>0$ for the radiation rate from cosmic string loops. In a second conjecture, we identify a candidate for the loop with the minimal radiation rate and suggest that $\gamma_{\min }^{0} \cong 39.003$.

PACS number(s): 98.80.Cq, 04.30.Db, 11.27.+d
\end{abstract}

Typeset using REVTEX 
Figure 5a

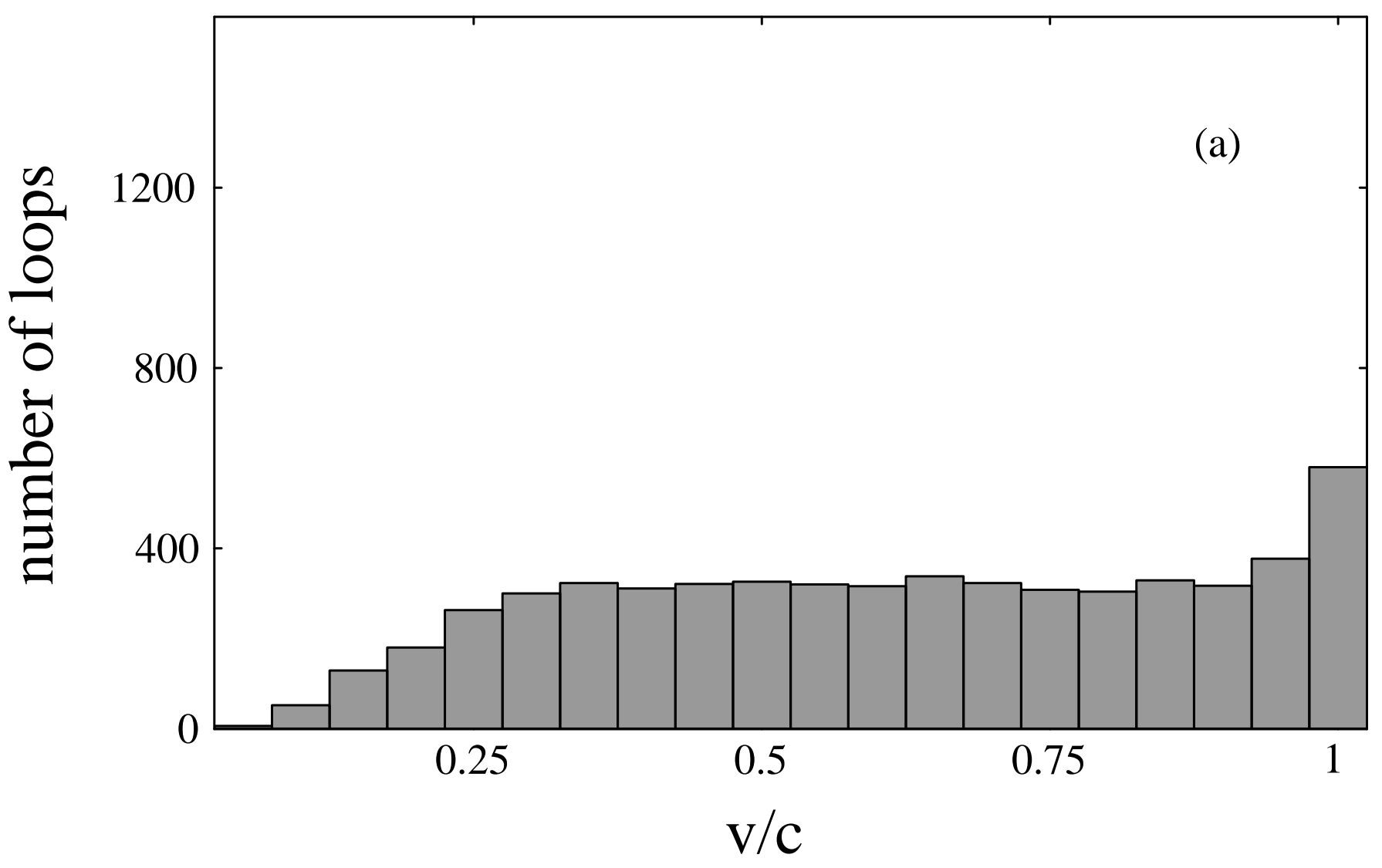


Figure $5 b$

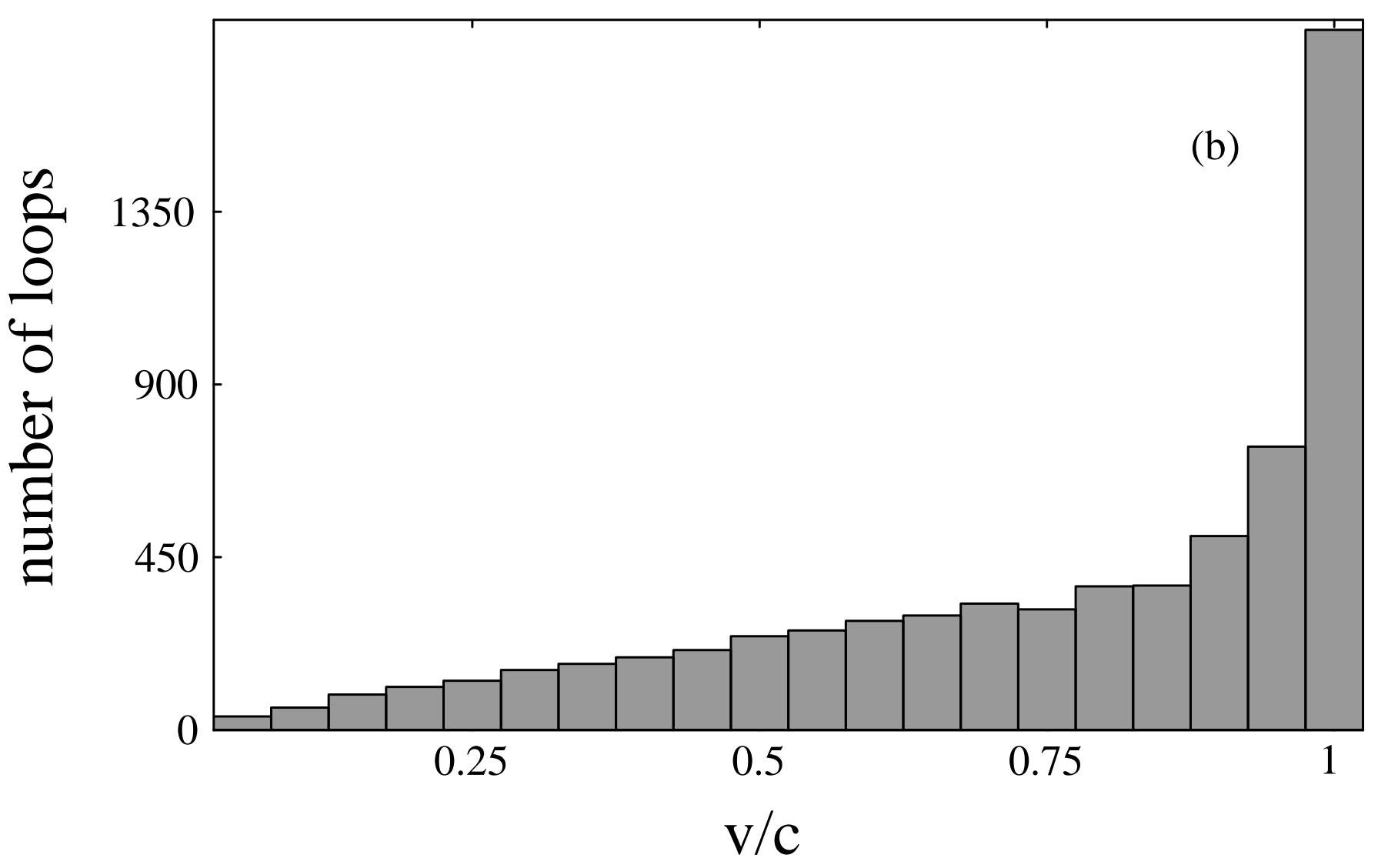


Figure 5c

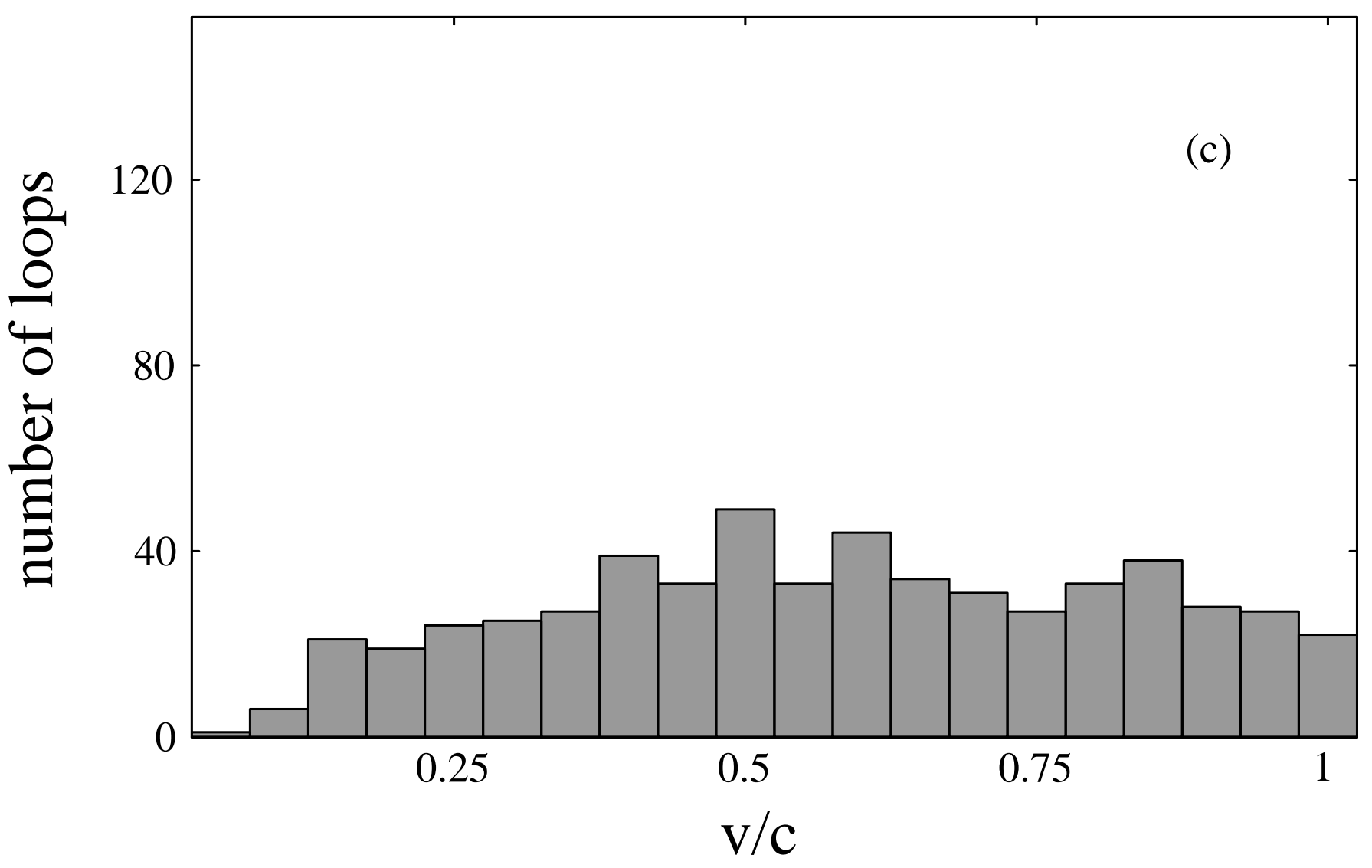


Figure 5d

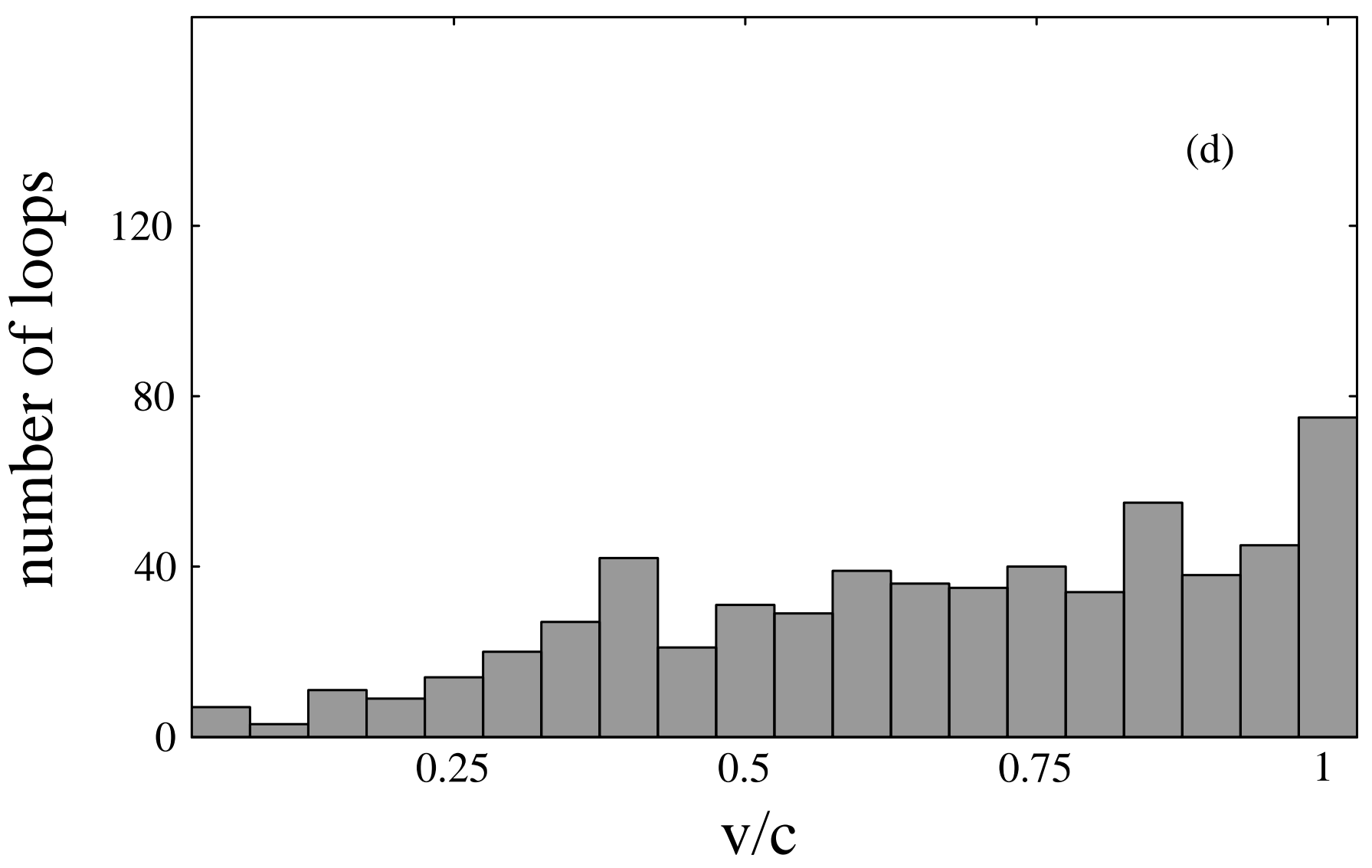


Figure 6a

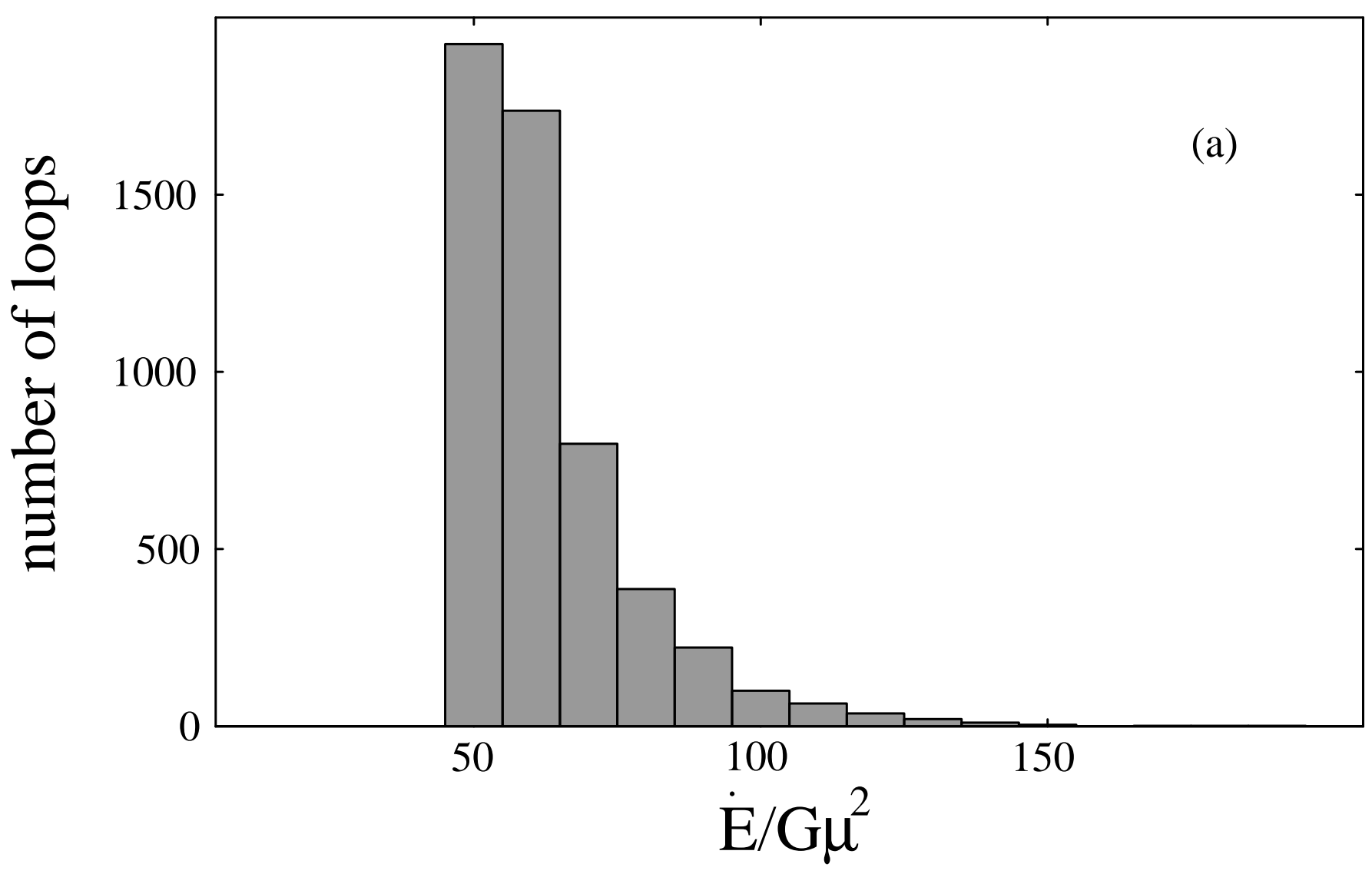


Figure $6 b$

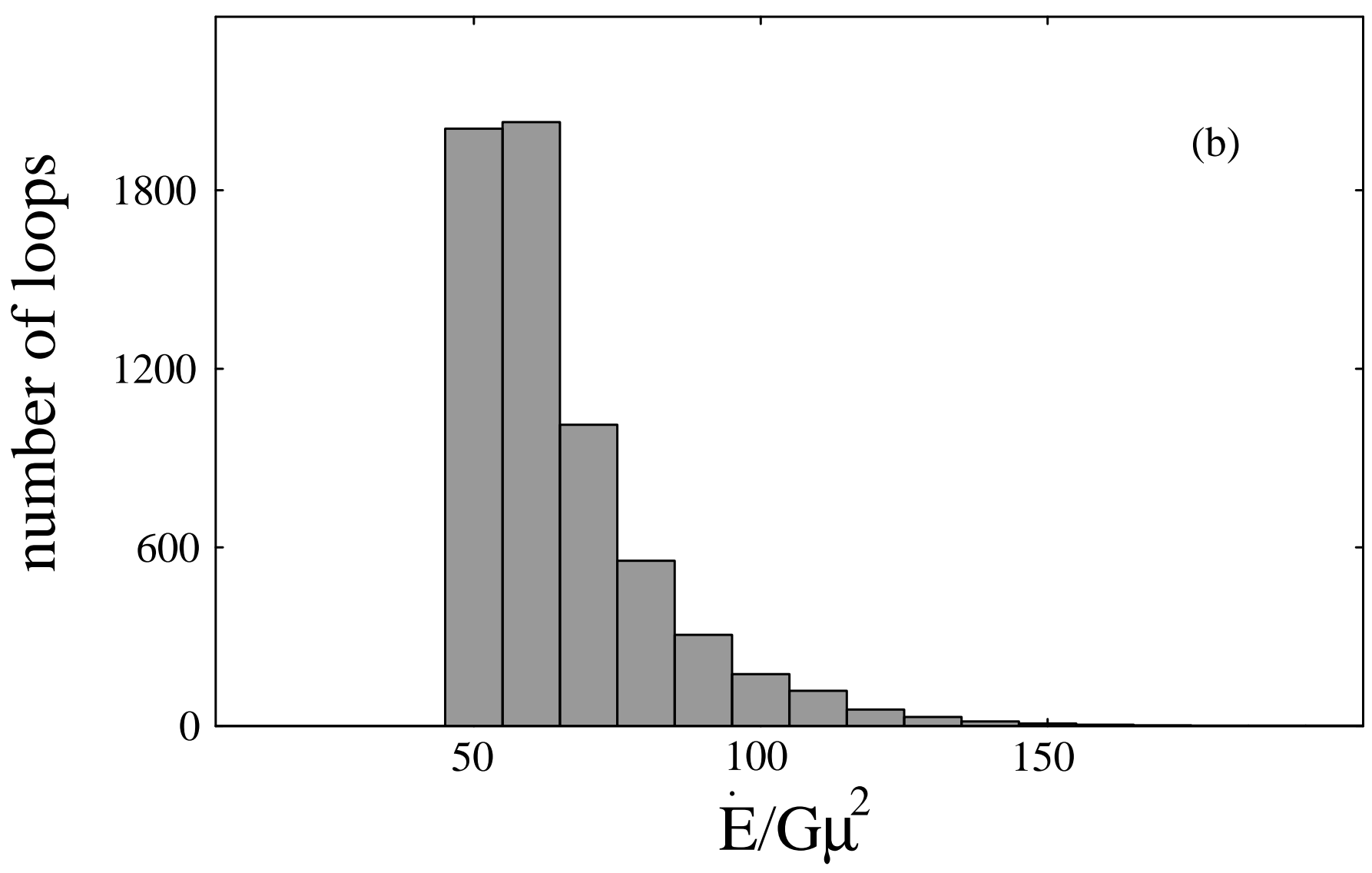


Figure 6c

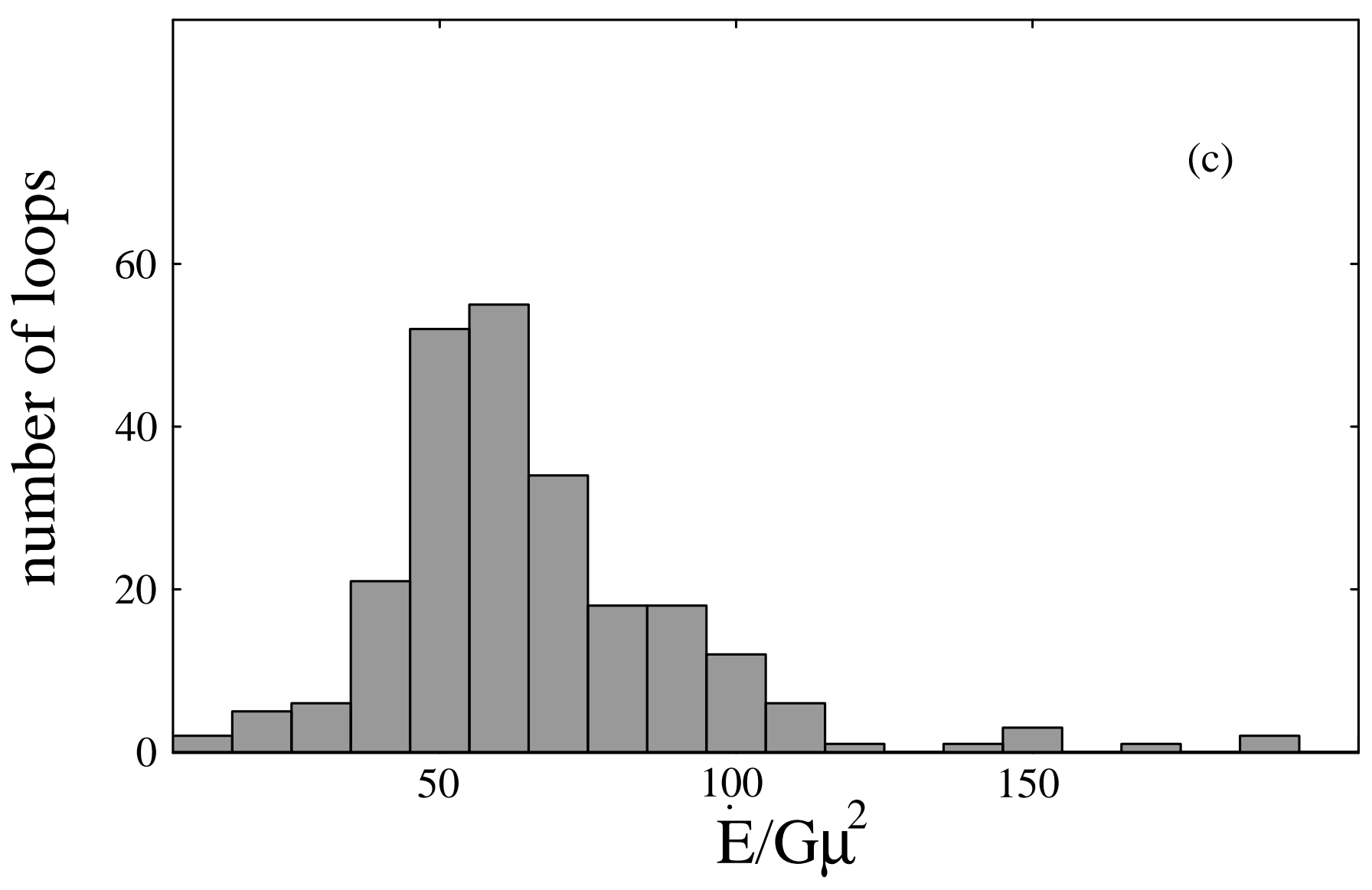


Figure 6d

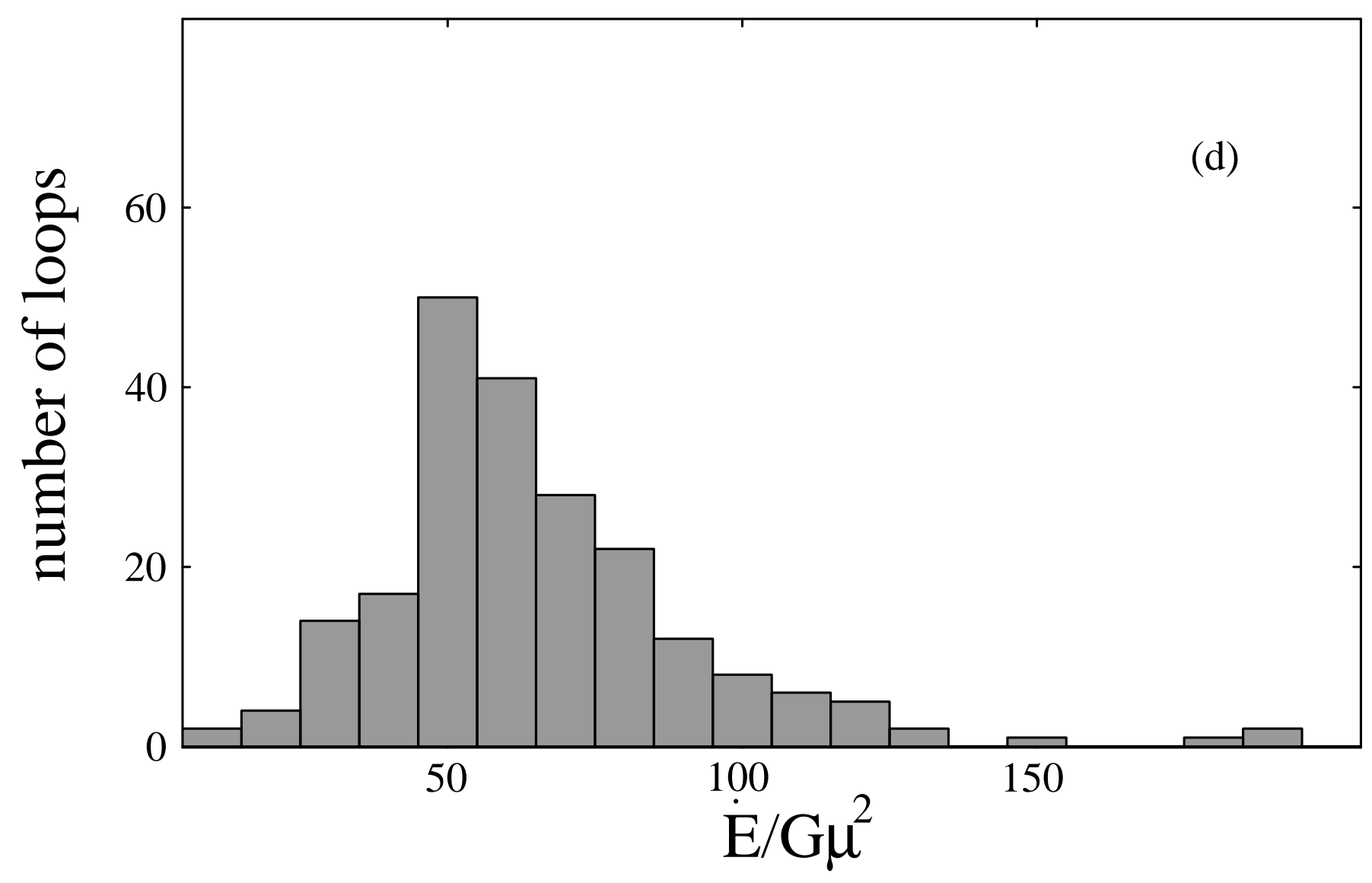


Figure $7 \mathrm{a}$

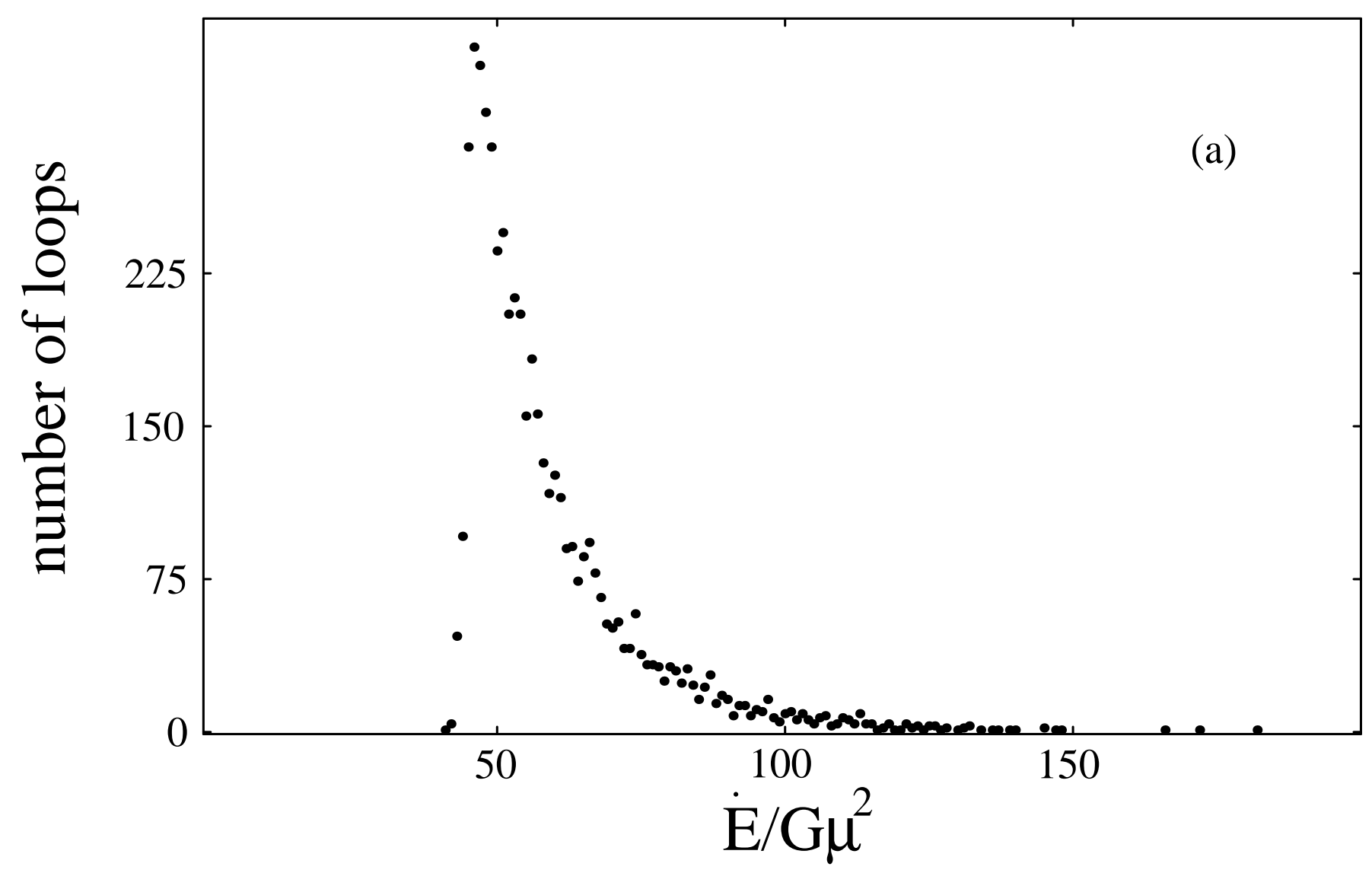


Figure $7 b$

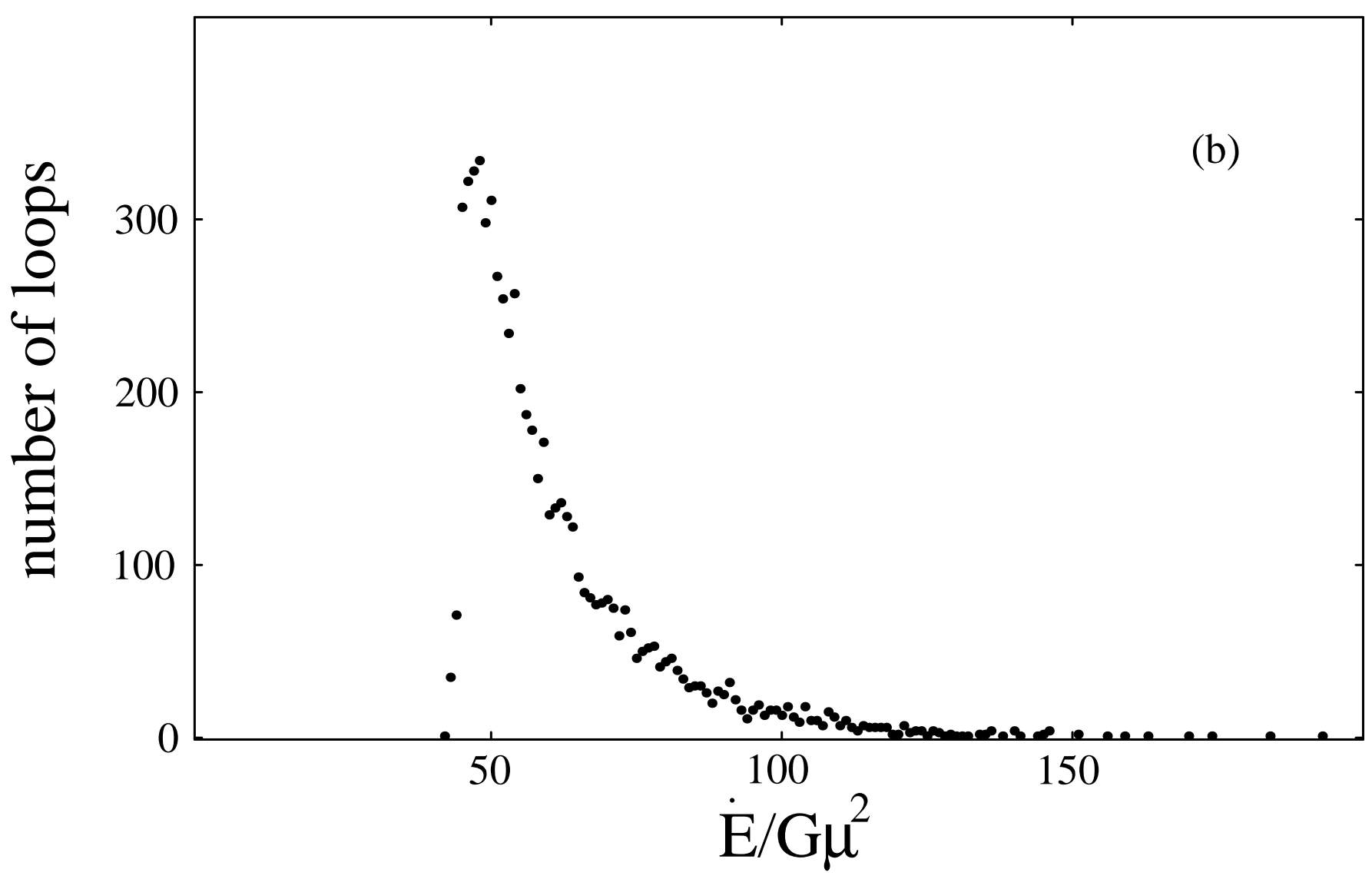


Figure 8a

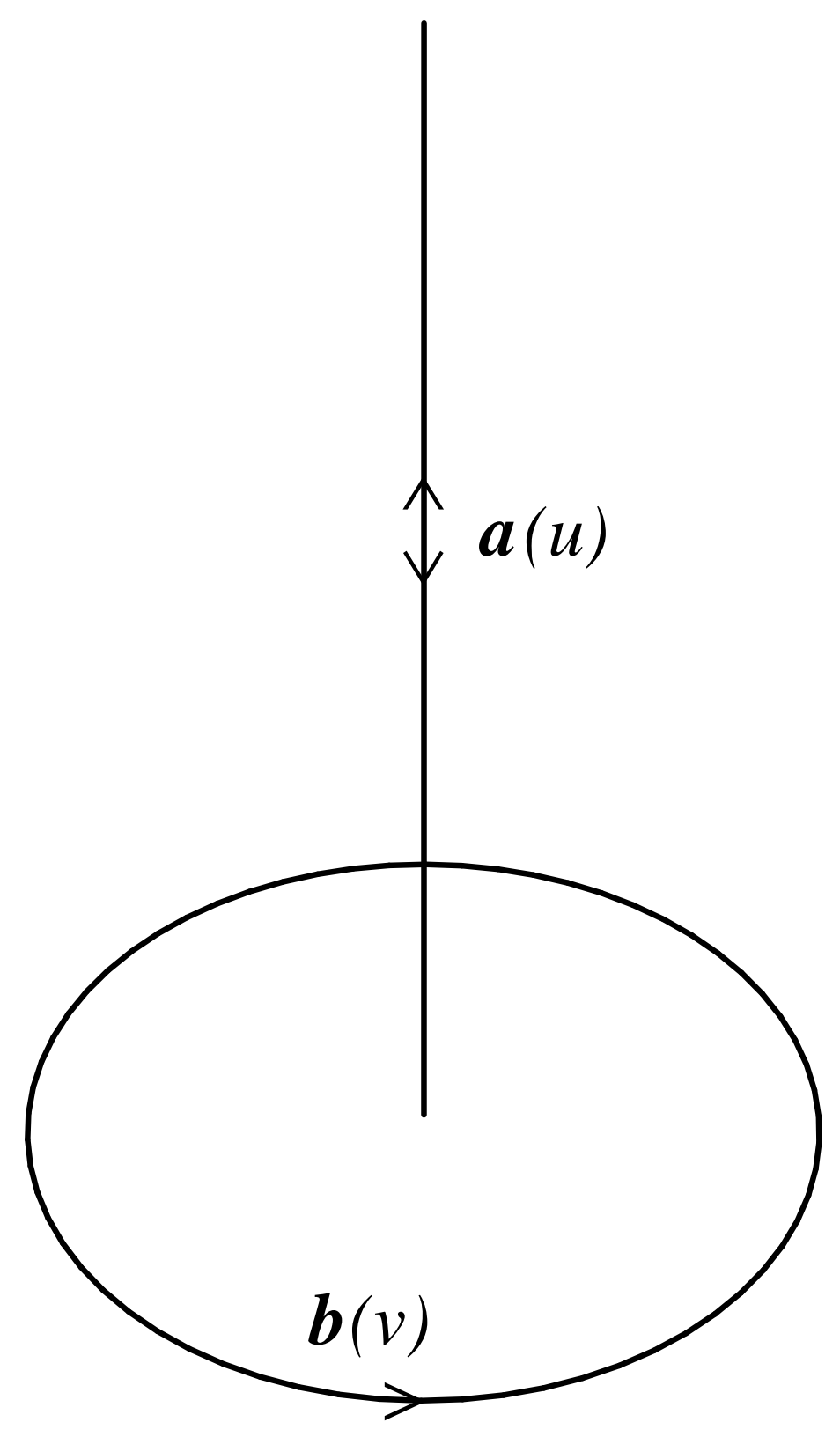

(a) 
Figure $8 b$

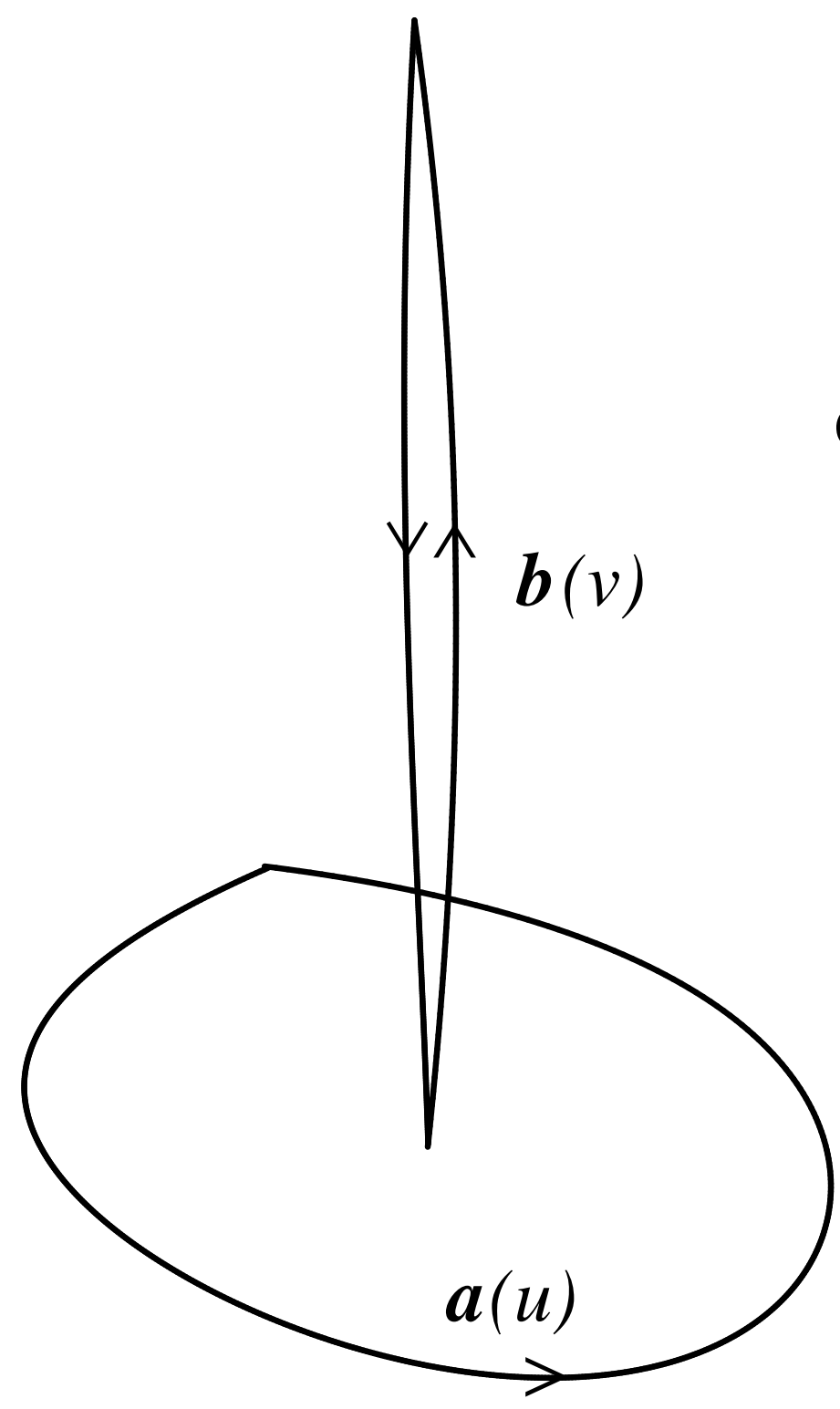

(b) 
Figure 8c

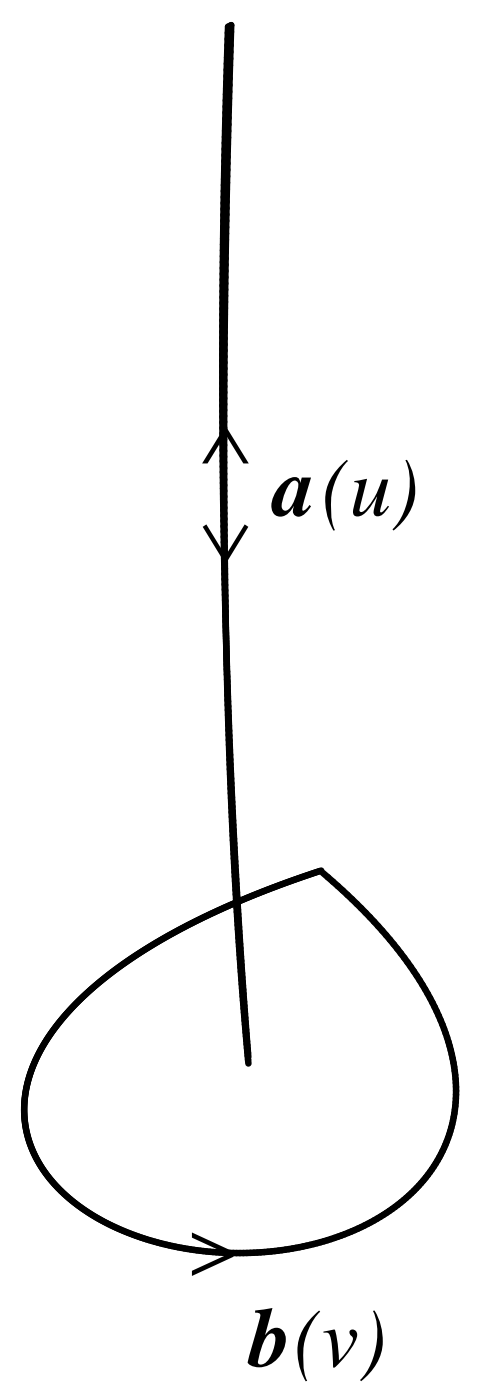

(c) 
Figure 8d

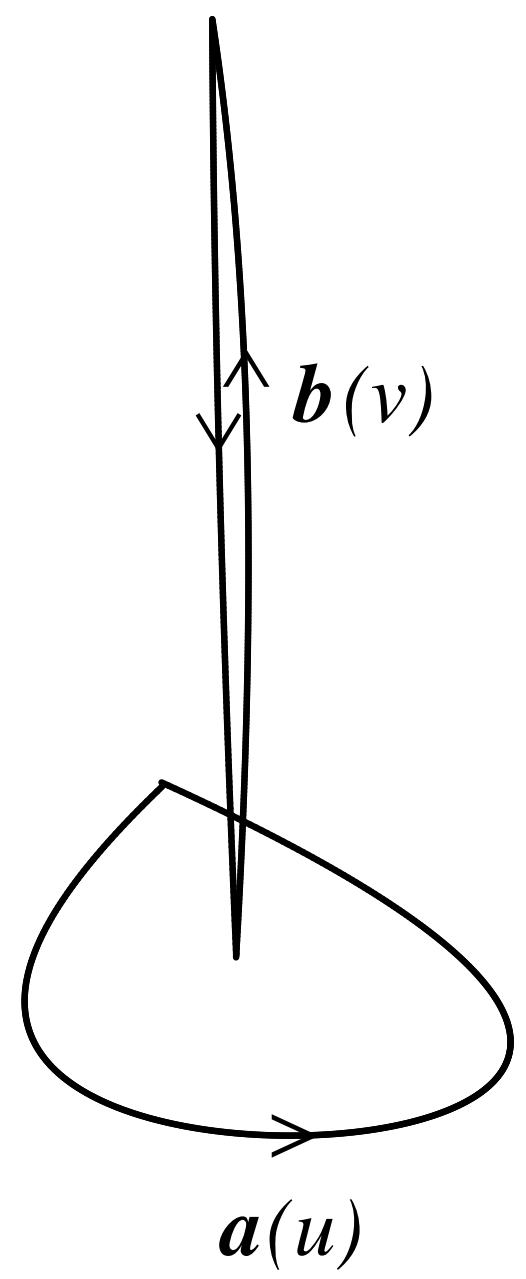


Figure 9a

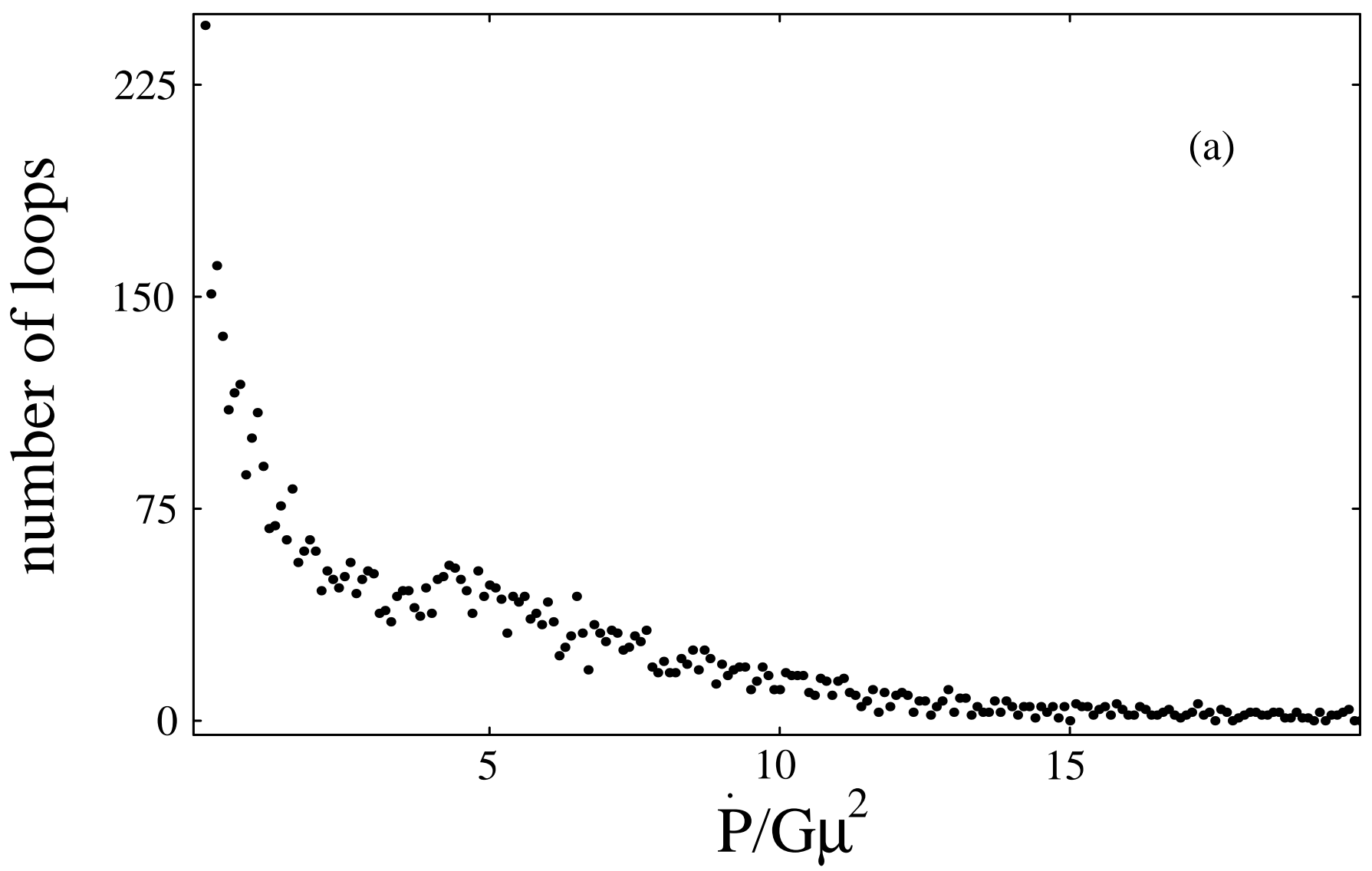


Figure $9 b$

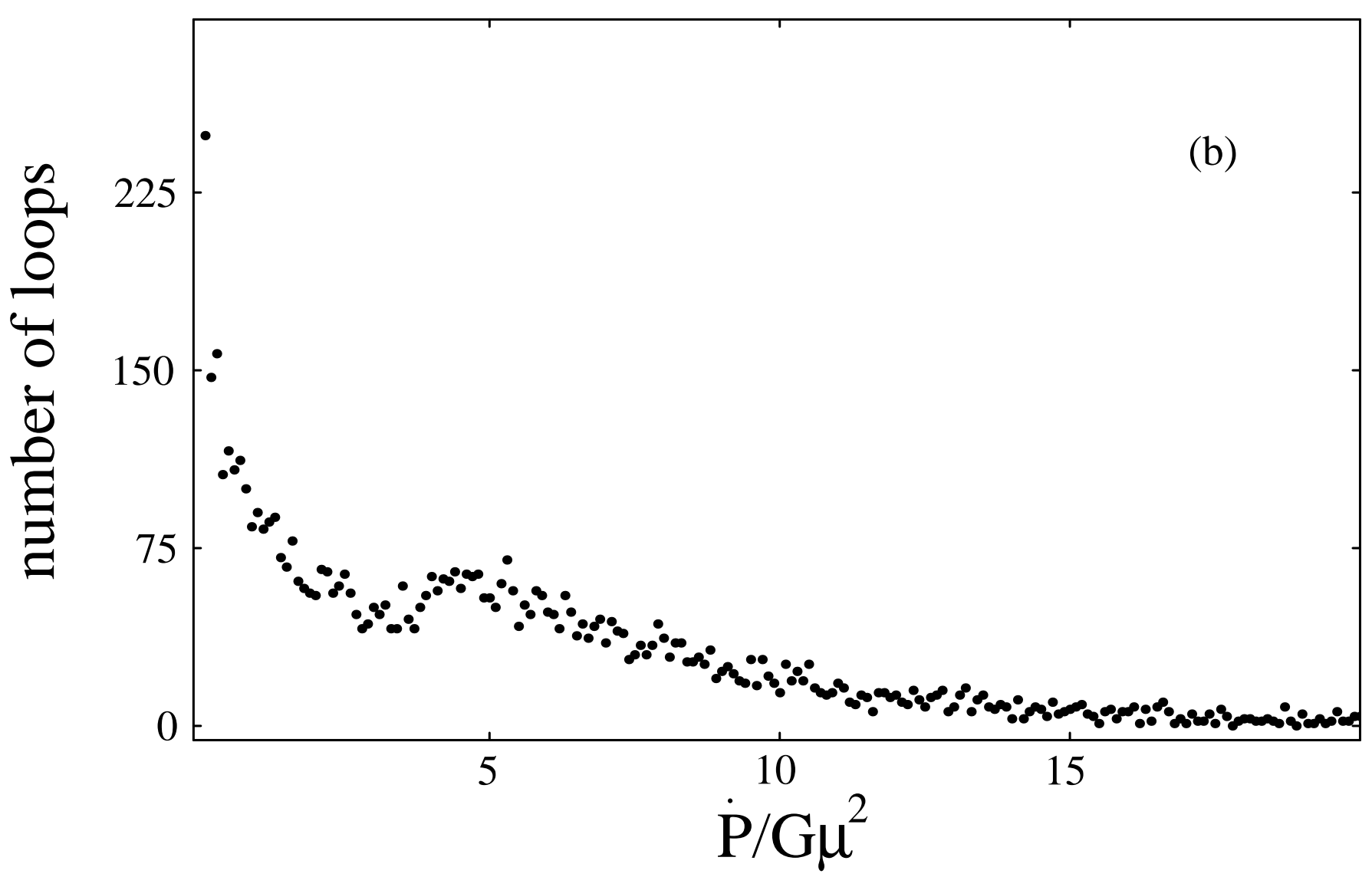

